\title{
The secretory senescence in otorhinolaryngology: principles of treatment
}

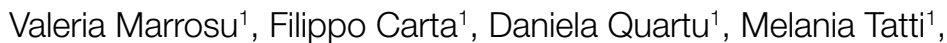
Cinzia Mariani', Daniele De Seta ${ }^{1}$, Roberto Puxeddu' ${ }^{1}$, Diletta Angeletti², Flaminia Campo ${ }^{2}$, Paolo Petrone ${ }^{3}$, Giacomo Spinato ${ }^{4}$, Alfonso Scarpa ${ }^{5}$, Gabriele Molteni', Giuditta Mannelli ${ }^{7}$, Pasquale Capasso ${ }^{8}$, Massimo Ralli², Valentina Casoli ${ }^{10}$, Francesco Antonio Salzano ${ }^{11}$, Stelio Antonio Mocella ${ }^{12}$, Francesco Barbara ${ }^{9}$, Salvatore Dadduzio ${ }^{9}$, Anna Berardi ${ }^{10}$, Carlo Berardi ${ }^{10}$

${ }^{1}$ Unit of Otorhinolaryngology, Department of Surgery, Azienda Ospedaliero-Universitaria di Cagliari, University of Cagliari, Italy; ${ }^{2}$ Department of Sense Organs, Sapienza University of Rome, Italy; ${ }^{3}$ Otolaryngology Department, Di Venere Hospital, Bari, Italy; ${ }^{4}$ Department of Neurosciences, Section of Otolaryngology and Regional Centre for Head and Neck Cancer, University of Padua, Treviso, Italy; ${ }^{5}$ Department of Medicine and Surgery, University of Salerno, Italy; ${ }^{6}$ Otorhinolaryngology Head and Neck Surgery, University Hospital of Verona Borgo Trento, Department of Surgery, Dentistry, Paediatrics and Gynaecology, University of Verona, Italy; ${ }^{7}$ Head and Neck Oncology and Robotic Surgery, Department of Experimental and Clinical Medicine, University of Florence, Italy; ${ }^{8}$ Otolaryngology Head and Neck Surgery Unit of "Azienda Ospedaliera di Rilievo Nazionale dei Colli, Ospedale Monaldi", Naples, Italy; ${ }^{9}$ Otolaryngology Unit, Mons. Dimiccoli Hospital, Barletta, Italy; ${ }^{10}$ Otolaryngology Department, Istituto Clinico Città Studi, Milan, Italy; ${ }^{11}$ Department of medicine, surgery and dentistry- university of Salerno , Italy; ${ }^{12}$ Day Clinic Medical Center Villafranca, Verona, Italy
\end{abstract}

Atrophy or hypofunction of the salivary gland because of aging, radiotherapy or disease causes hyposalivation and impairs the quality of life of patients by compromising mastication, swallowing and speech and by leading to a loss of taste. Moreover, hyposalivation exacerbates dental caries and induces periodontal disease, and oral candidiasis. Currently, no satisfactory therapies have been established to solve salivary hypofunction. Current treatment options for atrophy or hypofunction of the salivary glands in clinical practice are only symptomatic and include saliva substitutes and parasympathetic agonists, such as pilocarpine, to stimulate salivary flow. However, parasympathomimetics have systemic side effects, so different treatment options are necessary, and research has recently focused on this. The main strategies that have been proposed to restore salivary gland atrophy and hypofunction are gene therapy by gene activation/silencing during stem cell differentiation and by the use of viral vectors, such as adenoviruses; cell-based therapy with salivary gland cells, stem cells and non-salivary gland and/ or non-epithelial cells to regenerate damaged salivary gland cells; replacement with tissue bioengineering in which organoids from pluripotent stem cells are used in the development of organ replacement regenerative therapy. Remarkable progression in this research field has been made in the last decade, but a definitive therapy for salivary gland hypofunction has not been developed due to intrinsic challenges that come with each approach. However, with research efforts in the future, a range of precision medicine therapies may become available individualized to each patient.

Key words: secretory senescence, xerostomia, geriatric rhinitis, cell senescence, dysphagia, gene therapy, nutraceuticals
This is an open access article distributed in accordance with the CC-BY-NC-ND (Creative Commons Attribution-NonCommercial-NoDerivatives 4.0 International) license. The article can be used by giving appropriate credit and mentioning the license, but only for non-commercial purposes and only in the original version. For further information: https://creativecommons.org/licenses/by-nc-nd/4.0/deed.en
C Copyright by Società Italiana di Gerontologia e Geriatria (SIGG) 


\section{INTRODUCTION}

The secretory function of the upper aero-digestive tract has a key role in physiological processes such as eating and breathing. Aging is burdened by a functional alteration of the glandular function: in Otorhinolaryngology the main related scenarios are represented by xerostomia, rhinitis, and dry nose (DN). The secretory senescence deeply affects quality of life of elderly people: however, xerostomia, rhinitis and DN are frequently reported in patients burdened by several comorbidities and, as a consequence, these conditions are often neglected by clinicians and patients' relatives. Furthermore, these conditions are related with a damage of the upper aerodigestive tract mucosa and all the related functions of the oral cavity.

\section{XEROSTOMIA: CLINICAL FEATURES AND TREATMENT}

Among the different clinical pictures associated to the secretory senescence, those causing anomalies of the salivary secretion are the most life-quality affecting: the saliva has a determining role in the homeostasis of oral cavity because it helps in swallowing and speech, and prevents the mucosal damage thanks to its moistening and humidifying properties. Sialorrhea is another disfunction typical of aging, but it is generally associated to neurological disorders causing dysphagia or, at least, subclinical dysphagia, with consequent stagnant flow of saliva in the oral cavity and in the pharynx, without any correlation to alteration of the salivary secretion. Xerostomia is characterized by dry mouth, oral burning, sensation of a loss of or altered taste, difficulty with swallowing dry foods, and it can be associated to objective alterations of the mucosa such as diffuse erythema and dry fissured atrophy. Symptomatology of xerostomia is mainly related to the lack of the salivary electrolytes, peptides, glycoproteins, secretory immunoglobulins (type A), ammines and leucocytes which are key in the preservation of digestion, oral immune competence and balancing of oral bacterial flora; furthermore, since the saliva contrasts dental demineralization thanks to the buffer effect of the bicarbonates, chronic hyposalivation leads to impaired oral health, including a microbial shift that causes caries, periodontitis, loss of teeth, impaired masticatory function, and a higher risk of aspiration pneumonia.

Treatment of xerostomia can be divided in two main categories: systemic sialagogues and topical agents. Systemic sialagogues are mainly represented by Pilocarpine and Cevimeline. Pilocarpine is a non-selective muscarinic agonist with a mild beta-adrenergic ability.
Cevimeline is a parasympathomimetic and muscarinic agonist with high affinity for $\mathrm{M} 3$ receptors, that stimulate the salivary secretion. Pilocarpine dosage is usually $5 \mathrm{mg}$ three times a day, and Cevimeline is prescribed three times a day, $30 \mathrm{mg}$ per time; both drugs must be taken for at least 3 months ${ }^{1}$. These medications have demonstrated effectiveness ${ }^{2}$ in case of the presence of residual glandular tissue. However, a remarkable disadvantage of oral or systemic therapies is represented by the multiple-daily administration: elderly patients are generally less compliant to this kind of treatment because they usually already have a multiple drug background. Moreover, before any prescription of systemic sialagogues a strict evaluation must be undertaken: their intake is associated with several side effects such as: excessive sweating, cutaneous vasodilatation, emesis, nausea, diarrhea, persistent hiccup, bronchoconstriction, hypotension, bradycardia, increased urinary frequency, and vision problems ${ }^{3}$. Cevimeline, thanks to its higher selectivity, has fewer side effects: in literature the most frequent side effects complained are dizziness, sweating and urinary problems. These drugs are relatively contraindicated in asthmatic population and in beta-blockers users. Pilocarpine is also contraindicated in case of narrow-angle glaucoma and should be used with caution in individuals with chronic pulmonary disease. Some other systemic sialagogues that seem increase the salivary flow are Anethole and Yohimbine. Anethole is a cholagogue. It is recognized that anethole stimulates the parasympathetic nervous system and increases the secretion of acetylcholine, resulting in an increase in the signal transduction via its receptors, and consequently seems to increase the salivary flow ${ }^{4}$. Yohimbine is an alfa-2 blocker; in patients on antidepressant and neuroleptic medications it is described as xerostomia antagonist ${ }^{5}$. Topical agents can be classified in "salivary stimulants", "salivary substitutes", sugar-free chewing-gums, and candies. Given their user-friendliness, they can be considered the first-line approach in the management of xerostomia in the elderly. The $1 \%$ malic acid is a topic sialagogue which has been found to be effective but causes some enamel erosion ${ }^{6}$. Even though effective and particularly useful in elderly patients (they are not toxic even if ingested), salivary substitutes have a temporary effect and should be taken several times per day. They are oil and sugar-based (PEG-40, castor oil, xylitol, sucralose) and saliva substitutes resemble natural saliva, increasing salivary viscosity; therefore, saliva substitutes provide mucosal lubrification granting oral lubrification and comfort. In addition, given their content of Fluoride, they promote the enamel remineralization ${ }^{7}$. A more effective treatment of xerostomia should pay a particular attention to patient's therapies since dry mouth is a 
critical problem in elderly people with poly drugs: it is known that this problem can be a side effect of several medications such as neuroleptics, SSRI, FANS, Calcium channel blockers, sulfonylureas, opioids and so on (more than 1800 drugs and 80 classes, according to the literature) ${ }^{8}$. Equally important is the investigation of any underlying xerogenic comorbidities: rheumatoid pathologies (Sjogren syndrome, rheumatoid arthritis, scleroderma, mixed connective tissue disease) and deposit diseases (amyloidosis and sarcoidosis), cushing syndrome, diabetes mellitus, kidney disease, are associated with xerostomia. Some hygienic measures can be helpful. A good hydration is key to treat xerostomia, which is a critical factor in elderly patients. Air humidification at night-time, discontinuing irritant toothpastes (surfactants can be in fact aggravating factors in patients predisposed) and avoiding crunchy, spicy, and hard foods, can be effective approaches against xerostomia. Sugar-free gums and candies can be helping in case of residual glandular tissue ${ }^{9}$. Discontinuing medications that cause dry mouth or switching them to alternative agents should be considered if it is safe and necessary, and this can be crucial to the improvement of this symptom, which can be even settled. There are some less established treatments reported: the electrostimulation with portable devices or by the use of intraoral systems ${ }^{10}$, acupuncture ${ }^{11}$, hyperbaric therapy ${ }^{12}$, and low-dose laser therapy ${ }^{13}$.

\section{GERIATRIC RHINITIS: CLINICAL FEATURES AND TREATMENT}

In U.S., $40 \%$ of the elderly population complain nasal disfunctions such as nasal congestion, rhinitis, crusting, and dry nose (DN) ${ }^{14}$, that can be defined as geriatric rhinitis. The nasal function can be jeopardized by several predisposing factors associated with aging: nasal tip hanging, shortening of the columella, impairment of muco-ciliary clearance, loss of goblet cell population, and elastic fibers of basal membrane, enlargement of nasal cavity due to tissue atrophy, low mucosal sensitivity and dehydration due to the low water intake. Geriatric rhinitis can be characterized on the basis of the clinical presentation, and every different clinical condition requires a tailored therapy. Allergic and nonallergic hypersensitivity is characterized by an inflammatory reaction of the nasal mucosa after exposition to environmental factors; the incidence of these conditions decreases with age (less than 3 per 1000), and can be managed by the second-generation antihistamines (such as Loratadine and Cetirizine) which are much better tolerated than the first generation as they have fewer sedative effects. There is no study on the long-term therapeutic experience with Leukotrienes (Montelukast) for use in the elderly ${ }^{14}$. Decongestants (alfa-adrenergic agonists) are useful in case of hypersensitivity to reduce the acute oedema of the mucosa, but they must be managed carefully because in these patients their use can be easily stimulate the central nervous system causing several issues in case of heart disease, bladder disfunction, hypertension or neurovascular disease. Non-allergic rhinitis with eosinophilia (NARES) is typically associated with rhinorrhea and is successfully managed with steroids ${ }^{14}$. Vasomotor rhinitis in the elderly can be managed as the allergic rhinitis: a particular attention must be given using drugs such as Ipratropium, an anticholinergic drug with major antisecretory effect, for its potential side effects on cardiovascular, urinary and respiratory systems. Local dosage and maximum long-term administration time of Ipratropium is usually 1 to 2 puffs per nostril 204 times daily. The topical application has none of the systemic anticholinergic effects ${ }^{15}$. A widely recommended treatment in case of allergic, nonallergic and vasomotor rhinitis is represented by local steroids as their low incidence of side effects ${ }^{14}$. Drug-induced rhinitis can be caused by more than 400 medications such as adrenergic blockers (guanethidine), $\beta$-adrenergic blockers (propranolol), $\alpha$-adrenergic blockers (prazosin), vasodilators (hydralazine), and diuretics (hydrochlorothiazide), may cause nasal obstruction. Conjugated estrogens may also increase nasal airway resistance. Geriatric rhinitis can also be characterized by chronic and progressive atrophy of the mucosa of the nasal cavity alone, with reduction of the ciliary function and of the secretory cells: DN and crusting are therefore frequent consequences. A common complication of mucosal atrophy is the exposure and telangiectasia of submucosal vessels that can lead to frequent epistaxis, especially in case of some pharmacological predisposition (frequent use of blood thinners in elderly people). Therapeutic approach of DN can be tailored on the basis of its severity from some hygienic measures such as humidification and plenty hydration and, in case of mucosal changes of the anterior aspect of the nasal mucosa, oils and ointments only. DN with visible intranasal crust formation is the domain of nasal irrigation, which is better able to remove crusts than inhalation or sprays avoiding infections. Nasal rinses are recommended for the treatment of DN and their efficacy is provided by the cleaning mechanical effect which allows the removal of crusting, dense mucus, epithelial debris, irritant factors, and air polluting. In addition, nasal washing removes inflammatory mediators lying on the damaged mucosa and improves muco-ciliary clearance by increasing the nasal ciliary beat frequency (CBF). Several nasal sprays and nasal solutions (isotonic, hypertonic, and hypotonic) are 
available. Even though there are no diriment data on literature yet, isotonic solutions seem to improve symptoms preventing bacterial infections. Topical application of low-density oils (containing natural components such as soy oil, menthol, eucalyptus, and peanuts) is related to an improvement of CBF. A randomized study, comparing oils and saline solutions $\mathrm{NaCl}$-based, pointed out a higher moistening power of oily ointments; subjective sensation of DN seems to equally improve with both measures ${ }^{16}$. Moisturizing sprays can be adequate in case of DN sensation without any mucosal changes at nasal inspection. The rationale is that these medicaments can diminish the water loss. However, all these nasal topical agents, according to literature, improve symptoms of all geriatric rhinitis, such as nasal itching, pain, and irritation. Side effects reported can be nasal drip, nasal obstruction, and exacerbation of nasal itching. Main specific treatments reported in literature are summarized in Table I.

On our daily practice, an oncological background is often observed in patients with major secretory disfunctions, because of radiation therapy that causes irreversible changes on the irradiated mucosa. In these cases, topical agents and systemic hydration play a major role to minimize the actinic atrophy of the mucosa. Another critical point in the management of elderly patients complaining xerostomia and geriatric rhinitis is caused by the synergic effect of polypharmacy. For this reason, topical agents should be preferred.

\section{Gene theRAPy}

Activation of genes that improve regeneration has been recently studied, showing that treatment of mice with Alda-89, a selective aldehyde dehydrogenase 3 (ALDH3) activator, enriches for Kit+/CD90+ progenitors and increases proliferation of in vitro floating sphere assays (o salispheres). Also, gene therapy which involves transfer of a gene into cells to treat a disease or correct a cellular dysfunction has been widely studied.

Recently, pioneering gene therapy trials were completed by using the retroductal injection of an adenovirus that expressed a water channel (Aqp1) to transduce the remaining ductal epithelium to secrete fluid. Aquaporins are water channels necessary for transcellular water transport in salivary glands. Human Aqp1 gene transfer was demonstrated to be efficacious in nonhuman in vivo models. Recent results from phase I clinical trials showed no deaths, serious adverse events, or doselimiting toxicities. Objective and subjective restoration of salivary gland function was reported in some of the patients who received the AdhAQP1 vector ${ }^{17}$. These results are preliminary and further studies will be needed to confirm clinical efficacy and safety of gene therapy in restoration SGs function.

\section{Cell-based therapy}

Another therapeutic strategy is cell-based therapy. Epithelial stem/progenitor cells communicate with the surrounding niche, or microenvironment, that consists of vascular, stromal, and neuronal cells, to balance

Table I. Main specific treatments for secretory senescence mentioned in Literature ${ }^{1,2,4,5,10-13,15,16}$.

\begin{tabular}{|c|c|c|}
\hline $\begin{array}{l}\text { Secretory } \\
\text { disfunction }\end{array}$ & Systemic treatment & Topical treatment \\
\hline \multirow{3}{*}{ Xerostomia } & $\begin{array}{l}\text { Sialagogues: } \\
\text { - Pilocarpine hydrochloride, } 5 \mathrm{mg} / \text { day } \\
\text { - Cevimeline, } 30 \mathrm{mg} / \text { day }\end{array}$ & $\begin{array}{l}\text { Salivary stimulants } \\
\text { - Acid malic } 1 \%\end{array}$ \\
\hline & $\begin{array}{l}\text { Alfa } 2 \text { blockers } \\
\text { - Yohimbine } \\
\text { - Anethole trithione }\end{array}$ & $\begin{array}{l}\text { Salivary substitutes } \\
\text { - PEG-40 } \\
\text { - Castor oil } \\
\text { - Xylitol } \\
\text { - Sucralose }\end{array}$ \\
\hline & Hyperbaric therapy & $\begin{array}{l}\text { Local stimulators } \\
\text { - Acupuncture-like-transcutaneous-electrical-nerve- } \\
\text { stimulation } \\
\text { - Manual acupuncture } \\
\text { - Pulsed Ga-As laser }\end{array}$ \\
\hline \multirow[b]{2}{*}{$\begin{array}{l}\text { Geriatric } \\
\text { rhinitis }\end{array}$} & $\begin{array}{l}\text { Anti-inflammatory and anti-histaminergic drugs } \\
\text { - Prednisone } \\
\text { - Second-generation antihistamines }\end{array}$ & $\begin{array}{l}\text { Decongestants } \\
\text { - Ipratropium bromide } \\
\text { - Topical steroids }\end{array}$ \\
\hline & & $\begin{array}{l}\text { Nasal rinses and moisturizing sprays } \\
\text { - Saline solution (hypertonic, isotonic and hypotonic } \\
\text { solutions) } \\
\text { - Sodium cromoglycate given } \\
\text { - Pure sesame oil }\end{array}$ \\
\hline
\end{tabular}


stem cell processes and ensure tissue homeostasis. Several studies showed that stem/progenitor cells in salivary glands can restore salivation. Various kinds of cells have been used for transplantation in regenerative medicine. The stem cells themselves differentiate in the part of the organ where the function was restored. Cellbased therapies proposed to restore SG functions include autologous SG-derived epithelial stem/progenitor cells isolated from patients and transplanted to replace functionally damaged/lost cells and non-epithelial cells or their bioactive lysates used to trigger the paracrine regenerative effects on remaining glandular cells or to generate new glandular cells ${ }^{18}$.

The application of DNA labels to mark label-retaining quiescent cells, in vitro salispheres, and two-dimensional (2D) or three-dimensional (3D) cultures of both human and rodent $S G$ cells revealed the existence of multiple stem/progenitor-like cells in the SG. The first proof-of-concept study for transplanting autologous SG cells to increase salivary function was carried out in rodents and used epithelial cells expressing the cell surface receptor KIT (c-Kit, CD117). Studies revealed that various kinds of cytokines secreted by stem cells are related to the mechanisms of regeneration. These stem/progenitor cells can be identified and isolated; only 100-300 KIT+ cells were required to generate new acinar and ductal structures and to significantly improve organ function, this because mouse SGs contain cells with stem/progenitor properties that, when transplanted, could maintain themselves and differentiate toward multiple specialized SG cell types ${ }^{19}$. Further studies using transplantation of murine $\mathrm{KIT}+$ sub-populations (KIT+ CD24+, KIT+ CD49f+, KIT+ CD24+ CD49f+, $\mathrm{KIT}+\mathrm{CD} 24+\mathrm{SCA} 1+)$ showed that KIT+ cells located in the major ducts present with different levels of stem/ progenitor activity, and KIT1 CD24+ (CD49f+/SCA1+) cells are the most potent. Furthermore, KIT+ cells have potential for future cell therapy applications, particularly because they are present in human SGs and can be isolated and cultured ex vivo. In fact, using a variety of culture techniques, these studies showed that murine SG stem/progenitor cells are capable of in vitro expansion and differentiation toward parenchymal acinar- and duct-like cell lineages ${ }^{20}$.

Pringle et al. have recently further supported the clinical use of enriched KIT+ subpopulations. They presented data exploring the potential of human SG stem/progenitor cells, including their self-renewal and differentiation properties, and in vivo engraftment and functionality ${ }^{21}$. Interestingly, the nascent regenerative potential of murine salisphere-derived cells was enhanced upon selection of a CD24 ${ }^{\text {hi }} \mathrm{CD} 29^{\text {hi }}$ subset, associated with stem/progenitor cells in other tissues. These CD24hi $C$ D29hi salisphere cells showed pronounced self-renewal abilities indicated by high potential to form secondary salispheres and to differentiate into organoids containing both ductal and acinar cell lineages.

In vivo functionality of murine SG stem/progenitor cells has been repeatedly demonstrated, whereby as few as $100 \mathrm{CD} 117 / \mathrm{c}-\mathrm{kit}^{+}$cells, $300 \mathrm{CD} 24^{+} \mathrm{C}-\mathrm{Kit}^{+} \mathrm{Sca}-1^{+}$cells, or 10,000 culture-enriched $\mathrm{CD} 24^{\text {hi }} \mathrm{CD} 29^{\text {hi }}$ murine SG stem progenitor cells rescued hyposalivation in a mouse model.

Lombaert et al. proposed that combined KIT and FGFR2b signaling regulates epithelial progenitor expansion. They identified an interaction between KIT and FGFR2b signaling pathways that converges to upregulate a conserved group of FGFR2b-dependent TFs and expand distal progenitors. Their findings may have implications for regenerative medicine because they demonstrate that both KIT and FGFR2b signaling and communication among multiple cell types are necessary for organogenesis ${ }^{22,23}$.

Hitomi et al. focused on the differentiation of salivary gland cells using induced-pluripotent stem cells (iPS) in a microenvironment ${ }^{24}$.

Emmerson et al., using a combination of mouse genetics, ex vivo cultures, and human tissue explants, discovered that salivary acini are capable of regenerating after radiation in response to cholinergic activation through a progenitor cell-dependent mechanism ${ }^{25}$. The Authors demonstrated SOX2+ cells as progenitors in the adult salivary gland essential to the replenishment of acini with the capacity to repopulate the tissue after damage. They showed that cholinergic nerves play an important role in controlling SOX2-mediated acinar cell replacement during homeostasis, and that this neuronal influence can be replicated through addition of cholinergic mimetics to damaged tissue. Based on these data, targeting SOX2+ cells within the tissue, or by isolating and expanding these cells for transplantation and activation, regenerate the secretory units of salivary glands could be possible. However, additional experiments are needed to determine whether Sox2+ cells in the adult SMG are stem cells.

Recently, the transplantation of a bioengineered germ, with or without mesenchyme, was proposed as a promising strategy to replace SGs in a mouse model with SG defects. In fact, Tanaka et al. successfully regenerated the orthotopically functional salivary gland by using the transplantation of an induced salivary gland primordium (iSG) from mouse ESCs; they further demonstrated fully functional iSG by the recapitulation of the embryonic developmental process by the induction of an organforming field, transcription factors and maturation factors for the induction of salivary gland rudiment in vitro as an organ model. The iSGs orthotopically secreted saliva, which has salivary secretory proteins, by the 
reconstruction of neural network in vivo. This study provides the proof of concept of an organ induction and functional replacement of organoid induced from pluripotent stem cells for future organ replacement regenerative therapy ${ }^{26}$.

Togni et al. studied the generation of orthotopically functional salivary gland from embryonic stem cells, demonstrating that SGs orthotopically transplanted led to successful glandular development in vivo, showing connection of the new SG to the main duct, and correct formation of ductal and acinar structures. Noteworthy, the orthotopically engrafted SGs were functional in saliva secretion ${ }^{20,27}$.

There are many reports on the beneficial effects of non$S G$ and/or non-epithelial cells to regenerate damaged SGs. These studies include Bone Marrow (BM)-derived cells, BM-derived mesenchymal stem cells (MSC), human adipose-derived MSCs, SG-derived MSC-like cells, amniotic cells, embryonic stem cells (ESC), and iPS cells. A differentiation of BM-derived cells and MSCs into SG acinar cells is observed in vitro, while their actual contribution to epithelial differentiation in vivo is yet elusive. Their efficacy occurs via paracrine pro-survival/proliferative effects on remaining epithelial stem/progenitor cells and surrounding environmental cells. The transplantation of G-CSF/FLT3/SCF-mobilized BM-derived cells not only improved saliva production by inducing epithelial repair, but also increased micro vessel density, improving blood perfusion. Similarly, adipose-derived MSCs diminished acinar cell apoptosis and reduced fibrosis, and both BMMSC as SG-derived mesenchymal-like cells exerted immunosuppressive activities. The beneficial potential of these paracrine effects led to analyze the addition of the bioactive components ("soup") secreted by these adipose and BM-derived cells to repair SGs. The exact content of the bioactive components remains unclear, but several potential contributing signaling pathways have been identified. Moreover, intravenous "soup" administration may be useful to enhance saliva production as this delivery route appears to be as effective in rodents. However, it is unclear if the "soup" administration will be effective in all the patient. The "soup" strategy relies on the amount of remaining SG cells, so clinical successes will depend on the remaining cells that need paracrine stimulation ${ }^{28,29}$.

\section{BIOMATERIALS AND BIOENGINEERING}

Kawakami et al. examined methods of differentiation toward salivary gland cells from stem cells with only biomaterials. For biomaterials, they used mouse-derived early ES (mEES-6) as the cell source and fibroblasts were used as an inducer ${ }^{27}$. Their results seem promising to induce stem cell differentiation toward salivary gland cell by co-culture with salivary gland-derived fibroblasts in order to start the generation of salivary gland tissue and to finally restore the gland function by transplantation of the differentiated salivary glands cells in vivo.

Next-generation regenerative therapy is organ replacement regenerative therapy. Organoids from pluripotent stem cells are used in the development of organ replacement regenerative therapy by recapitulating the organogenesis. The process of organogenesis is regulated by morphogen signaling and transcriptional networks even if the transcription factors involved remain unknown ${ }^{26}$. Organoid studies demonstrated that various organs can be regenerated by the recapitulation of the early developmental process of organogenesis from PSCs in vitro. These organoids have a complex organ structure and function by using complex mini-organs. To date, it is still unexplored whether the organoids or the regenerated primordia will fully function after orthotopic transplantation, with a sufficient size and correct morphology in vivo.

SG tissue engineering requires three essential components cell-cell contacts, cell contacts with extracellular matrix (ECM) proteins, and a biocompatible and biodegradable $3 \mathrm{D}$ scaffold to hold these components together.

Many scaffolds have been proposed, such as porous and biologic scaffold (e.g., collagen, fibrin, silk, chitosan, alginate, hyaluronic acid) or synthetic biocompatible biomaterials (e.g., poly-glycolic acid, poly-lactic acid, poly lactic-co-glycolic acid, and polyethylene glycol), and/or mixture of both. Their characteristics of biodegradability, porosity, stiffness, and strength are necessary to promote cell adhesion, migration, and/or differentiation. Ideally, engineered scaffolds should structurally and functionally resemble the native SG ECM architecture.

Recent advances have also been focused on the development of more functional organoids. These can then be seeded with cells to direct differentiation and branching. A current challenge remains to let bioengineered tissues grow in size and properly integrate with remaining cells in the transplanted area. Whether a similar reconnection with the remaining duct can be obtained in humans remains to be determined.

Previous studies have shown that paracrine and autocrine morphogenic gradients control branching morphogenesis, and a number of models propose the existence of iterative positive and negative unidirectional cues between the epithelium and mesenchyme or within the epithelial lineage. These studies provided valuable insights into organogenesis but focused on communication from a single cell type. Progenitors in vivo must interpret signals from multiple cell types 
in their surrounding microenvironment during organogenesis.

Recent advances showed a bioengineered gland from fetal epithelium and mesenchyme transplanted into an adult mouse to form a new functional gland in the adult microenvironment. This bioengineered gland contained a variety of fetal cells, including progenitors of epithelial, mesenchymal, endothelial and neuronal cells. The newgland reconnected with the ductal system and was able to secrete saliva, ensuring protection of the oral cavity from bacteria, and restoration of normal swallowing ${ }^{30}$. The aim now will be to use induced pluripotent stem cells or adult salivary progenitors to form a bioengineered rudiment that grows into a functional gland in the adult microenvironment. It is necessary to understand the mechanisms by which progenitors expand and how they communicate with other cell types in order to regenerate or reengineer the branched architecture of epithelial organs. Regenerative therapy represents a fundamental approach for treating patients who experience organ dysfunction as the result of disease, injury or ageing ${ }^{28}$. The goal of regenerative medicine is to restore gland function in patients who suffer from irreversible loss of salivary gland function for pathologies, irradiation, or ageing.

\section{CELL SENESCENCE AND NUTRACEUTICALS}

Recent studies have shown that some cells experience irreversible stunting and become the cornerstone of many age-related diseases. This occurs through the activation of tumor suppressors such as p53 and p16ink4a caused by shortening of telomeres, mitogenic signals, protein aggregation and others.

From the beginning of this cellular modification process, senescence can take days to weeks to become stable and irreversible and undergoes gradual reinforcement thanks to cyclic intracellular signals. In addition to being metabolically active, senescent cells are resistant to apoptosis and can secrete pro-inflammatory cytokines, chemokines. and proteases, thus becoming cells associated with secretory phenotype (senescence-associated-secretory-phenotype, SASP). These play an important role in the genesis of that slow-inflammation condition that accompanies humans in age-related frailty.

The selective elimination of SASP cells and/or their effects would lead to a decrease in chronic phlogosis and is currently one of the objectives of anti-aging medicine ${ }^{31-33}$.

\section{NUTRACEUTICALS IN THE "FIGHT AGAINST SASP" 34}

Western medical culture is deeply linked to drug treatments based on the search for increasingly sophisticated synthetic molecules. In this well-radicalized clinical practice, a new concept of cure and above all prevention is making its way, the possibility of using molecules already present in nature which, alongside

Table II. Main nutraceutical compounds and their clinical effects.

\begin{tabular}{|l|l|}
\hline $\begin{array}{l}\text { Harpagopuytum Procumber } \\
\text { (devil's claw) }\end{array}$ & $\begin{array}{l}\text { It inhibits the release of TNF-alpha, IL6, IL1beta and inhibits proinflammatory genes. It has a dose- } \\
\text { dependent function and is very effective in both acute and subacute inflammation }\end{array}$ \\
\hline Boswella Serrata & $\begin{array}{l}\text { Numerous studies have shown efficacy in reducing the inflammatory state in patients suffering from } \\
\text { chronic pathologies such as ulcerative rectocolitis, bronchial asthma, rheumatoid arthritis }\end{array}$ \\
\hline Bromelain (pineapple comosus) & $\begin{array}{l}\text { It contains many proteolytic enzymes that increase serum fibrinolytic activity, decrease the levels of } \\
\text { plasma fibrinogen and bradykinin leading to a reduction in vascular permeability, edema and pain }\end{array}$ \\
\hline Turmeric Longa (turmeric) & Inhibits the metabolism of arachidonic acid, COX-2, lipoxygenase, interleukins and TNF-alpha \\
\hline Olea Europea (olive oil) & $\begin{array}{l}\text { Effective on chronic low-grade diseases thanks to the modulation of some cytokines and the inhibition of } \\
\text { COX-2 and iNOS and antioxidant effects on the endothelium }\end{array}$ \\
\hline Grapes (Vitis Vinifera) & $\begin{array}{l}\text { It contains polyphenols that through various action mechanisms perform both a pro-inflammatory and } \\
\text { anti-inflammatory action. They inhibit the synthesis and release of proinflammatory mediators, inhibit } \\
\text { COX-1 and COX-2 and other transcription factors }\end{array}$ \\
\hline Cherry (prunus ceraus) & $\begin{array}{l}\text { Cherry juice contains large quantities of anthocyanins that have antioxidant and anti-inflammatory } \\
\text { properties, by inhibiting TNF-alpha and COX-2. } \\
\text { Large quantities of anthocyanins are also found in cranberries and strawberries }\end{array}$ \\
\hline Role of omega 3 fatty acids & $\begin{array}{l}\text { At high doses they attenuate the inflammatory gene expression of the adipose tissue thus counteracting } \\
\text { disorders such as obesity and diabetes. They are also used in the treatment of joint pain }\end{array}$ \\
\hline Role of probiotics & $\begin{array}{l}\text { The food and agriculture organization/world health organization defines probiotics as "living organisms } \\
\text { which, when administered in adequate quantities, confer a benefit on the health of the host". Their } \\
\text { fundamental role has been demonstrated in improving components of the immune response such as } \\
\text { phagocytosis, the activity of natural killer cells and the production of IgA }\end{array}$ \\
\hline
\end{tabular}


traditional therapies, projects current medicine towards an improvement of the body response to an inflammatory condition that can become chronic over the years (Tab. II).

In the light of this, it is essential to introduce 2 concepts around which the basis of nutraceuticals revolves: epigenetics and caloric restriction. Epigenetics is considered as the set of information (mitotically and/or meiotically inheritable) in addition to the genetic information that must be present to control gene expression and allow development and differentiation of a complex organism, without DNA modification. The drastic reduction, without incurring malnutrition, of caloric intake in humans is referred to as caloric restriction $(C L)$ and has been shown to constitute a kind of slight stress that contributes to increasing life expectancy by activating the hormesis process (together of stress that positively affect the well-being of the individual). $C L$ is able of reducing risk factors for age-related diseases because increases mitochondrial biogenesis, counteracts endocrine-senescence, reduces DNA damage and improves gene expression, lowers the inflammatory state and stimulates autophagy.

Thus, let us see how it is not only about genes, diet or organic pathology, but how it is necessary to consider a systemic framework of the individual and the environment that surrounds him to get to a personalized medicine. Being a latent condition of chronic inflammation as a cause of human aging, it is evident that nutraceuticals associated with a correct diet can counteract the effects of SASP, with the aid of substances that can intervene on the inflammatory phenomena promoted by this type of senescent cells ${ }^{35}$.

Nutraceuticals have many molecules capable of acting by decreasing the production of inflammation mediators, antioxidant agents and improving the intestinal barrier function. It can therefore be said that nutraceuticals play a very important role in the inflammatory response and in the prevention of its chronicization. With the aim of increasing that time period in which chronological aging does not correspond to an aging of health and to a physical and cognitive deterioration, we can think of counteracting some chronic inflammatory processes that arise due to the secretory pattern of the cells that have gone in a state of senescence. Nutraceuticals could play a key role as they are simple to use and without particular contraindications in repeated or long-term administrations, just think of the regular introduction of certain substances in the diet and in daily nutrition.

While waiting to be able to perform in vivo studies on the non-harmful elimination of senescent cells, other studies on the therapeutic and preventive use of molecules such as omega3, boswella, turmeric and cherry can be carried out in populations divided by age group.
A good prevention of the non-microbial chronic phlogosis condition would lead to a decrease in the years in which the patient experiences disability and fragility and an extension of the life lived at reduced health costs. In the otolaryngologic field, some diseases that invalidate and compromise the defenses of VAS such as chronic laryngitis and chronic rhinitis could be better controlled, and their onset could be delayed.

\section{CONCLUSIONS}

Remarkable progression has been made in the last decade, but a definitive therapy for SG hypofunction has not been developed due to intrinsic challenges that come with each approach. Moreover, potential differences in development and/or regenerative strategies between the parotid submandibular and sublingual glands need to be considered for future clinical translations.

With the aim of increasing that time period in which chronological aging does not correspond to an aging of health and to a physical and cognitive deterioration, we can think of counteracting some chronic inflammatory processes that arise due to the secretory pattern of the cells that have gone in a state of senescence. Nutraceuticals could play a key role as they are simple to use and without particular contraindications in repeated or long-term administrations, just think of the regular introduction of certain substances in the diet and in daily nutrition.

The management of secretory senescence is office-based and does not require any hospitalization. Physicians should not underestimate symptoms related to the secretory senescence, paying attention to patient comorbidities, and regular evaluations are necessary to assess the best tailored treatment based on patient needs.

\section{References}

1 Aframian D, Helcer M, Livni D, et al. Pilocarpine treatment in a mixed cohort of xerostomic patients. Oral Dis 2007;13:88-92. https://doi.org/10.1111/j.16010825.2006.01252.x

2 Braga M, Tarzia O, Bergamaschi C, et al. Comparison of the effects of pilocarpine and cevimeline on salivary flow. Int J Dent Hyg 2009;7:126-30. https://doi.org/10.1111/ j.1601-5037.2008.00326.x

3 Villa A, Connell CL, Abati SJT, et al. Diagnosis and management of xerostomia and hyposalivation. Ther Clin Risk Manag 2015;11:45. https://doi.org/10.2147/TCRM.S76282

4 Hamada T, Nakane T, Kimura T, et al. Treatment of xerostomia with the bile secretion-stimulating drug anethole trithione: a clinical trial. Am J Med Sci 1999;318:146-51 https://doi.org/10.1097/00000441-199909000-00009 
5 Bagheri H, Schmitt L, Berlan M, et al. A comparative study of the effects of yohimbine and anetholtrithione on salivary secretion in depressed patients treated with psychotropic drugs. Eur J Clin Pharmacol 1997;52:339-42. https://doi. org/10.1007/s002280050298.

6 Gómez-Moreno G, Guardia J, Aguilar-Salvatierra A, et al. Effectiveness of malic acid $1 \%$ in patients with xerostomia induced by antihypertensive drugs 2013;18: e49.

7 Plemons JM, Al-Hashimi I, and Marek CLJTJotADA, Managing xerostomia and salivary gland hypofunction: executive summary of a report from the American Dental Association Council on Scientific Affairs. J Am Dent Assoc 2014;145:867-73. https://doi.org/10.14219/jada.2014.44

8 Singh ML and Papas AJDC. Oral implications of polypharmacy in the elderly. Dent Clin North Am 2014;58:783-96. https://doi.org/10.1016/j.cden.2014.07.004

9 Furness S, Worthington HV, Bryan G, et al. Interventions for the management of dry mouth: topical therapies. Cochrane Database Syst Rev 2011:CD008934. https:// doi.org/10.1002/14651858.CD008934.pub2

10 Wong RK, James JL, Sagar S, et al. Phase 2 results from Radiation Therapy Oncology Group Study 0537:a phase 2/3 study comparing acupuncture-like transcutaneous electrical nerve stimulation versus pilocarpine in treating early radiation-induced xerostomia. Cancer 2012;118:4244-52. https://doi.org/10.1002/cncr.27382

11 Cho JH, Chung WK, Kang W, et al. Manual acupuncture improved quality of life in cancer patients with radiationinduced xerostomia. J Altern Complement Med 2008;14:523-6. https://doi.org/10.1089/acm.2007.0793

12 Teguh DN, Levendag PC, Noever I, et al. Early hyperbaric oxygen therapy for reducing radiotherapy side effects: early results of a randomized trial in oropharyngeal and nasopharyngeal cancer. Int $J$ Radiat Oncol Biol Phys 2009;75:711-6. https://doi.org/10.1016/j. ijrobp.2008.11.056

13 Lončar B, Mravak Stipetić M, Baričević M, et al. The effect of low-level laser therapy on salivary glands in patients with xerostomia. Med Laser Surg 2011;29:171-5.

1414 Slavin RGJA, Asthma and Immunology C. Treating rhinitis in the older population: special considerations. Allergy Asthma Clin Immunol 2009;5:9. https://doi. org/10.1186/1710-1492-5-9

15 Tan R, Corren JJD. Optimum treatment of rhinitis in the elderly. Drugs Aging 1995;7:168-75. https://doi. org/10.2165/00002512-199507030-00002

16 Johnsen J, Bratt B-M, Michel-Barron O, et al. Pure sesame oil vs isotonic sodium chloride solution as treatment for dry nasal mucosa. Arch Otolaryngol Head Neck Surg 2001;127:1353-6.

17 Holmberg KV, Hoffman MP, Anatomy, biogenesis and regeneration of salivary glands, in saliva: secretion and functions. Karger Publishers 2014, pp. 1-13.

18 Lin C-Y, Chang F-H, Chen C-Y, et al. Cell therapy for salivary gland regeneration. J Dent Res 2011;90:341-6. https://doi.org/10.1177/0022034510386374

19 Nanduri LS, Lombaert IM, van der Zwaag M, et al.
Salisphere derived c-Kit+ cell transplantation restores tissue homeostasis in irradiated salivary gland. Radiother Oncol 2013;108:458-63. https://doi.org/10.1016/j.radonc.2013.05.020

20 Togni L, Mascitti M, Santarelli A, et al. Unusual conditions impairing saliva secretion: developmental anomalies of salivary glands. Front Physiol 2019;10:855 https://doi. org/10.3389/fphys.2019.00855

21 Pringle S, Maimets $M$, van der Zwaag $M$, et al. Human salivary gland stem cells functionally restore radiation damaged salivary glands. Stem Cells 2016;34:640-52. https:// doi.org/10.1002/stem.2278

22 Lombaert I, Movahednia MM, Adine C, et al. Concise review: salivary gland regeneration: therapeutic approaches from stem cells to tissue organoids. Stem Cells 2017;35:97-105. https://doi.org/10.1002/stem.2455

23 Lombaert IM, Abrams SR, Li L, et al. Combined KIT and FGFR2b signaling regulates epithelial progenitor expansion during organogenesis. Stem Cell Reports 2013;1:604-19. https://doi.org/10.1016/j.stemcr.2013.10.013

24 Ono H, Obana A, Usami Y, et al. Regenerating salivary glands in the microenvironment of induced pluripotent stem cells. Biomed Res Int 2015;2015:293570. https:// doi.org/10.1155/2015/293570

25 Emmerson E, May AJ, Berthoin L, et al. Salivary glands regenerate after radiation injury through SOX2-Mediated secretory cell replacement. EMBO Mol Med 2018;10:e8051.

26 Tanaka J, Ogawa M, Hojo H, et al. Generation of orthotopically functional salivary gland from embryonic stem cells. Nat Commun 2018; 9:1-13. https://doi.org/10.1038/ s41467-018-06469-7

27 Kawakami M, Ishikawa $\mathrm{H}$, Tachibana $\mathrm{T}$, et al. Functional transplantation of salivary gland cells differentiated from mouse early ES cells in vitro. Hum Cell 2013;26:80-90. https://doi.org/10.1007/s13577-013-0061-z

28 Ogawa M, Oshima M, Imamura A, et al. Functional salivary gland regeneration by transplantation of a bioengineered organ germ. Nat Commun 2013;4:1-10. https://doi. org/10.1038/ncomms3498

29 Aure M, Arany S, Ovitt CJ. Salivary glands: stem cells, selfduplication, or both? J Dent Res 2015;94:1502-7.

30 Patel VN Hoffman MP. Salivary gland development: a template for regeneration. Sem Cell Develop Biol 2014.

31 Galimberti D. La medicina dell'aging e dell'anti-aging. Milano: Elsevier Italia 2016.

32 Galimberti D, Gidaro G, Calabrese V, et al. Nutrigenomica ed epigenetica: dalla biologia alla clinica. Milano: Edra 2017.

33 Cicero AFG, Trattato italiano di nutraceutica clinica. Milano: Scripta Manent 2017.

34 Nakagami HJG and International G, Cellular senescence and senescence-associated $T$ cells as a potential therapeutic target. Geriatr Gerontolog Int 2020;20:97-100. https://doi.org/10.1111/ggi.13851.

35 Tchkonia T, Zhu Y, van Deursen J, et al. Cellular senescence and the senescent secretory phenotype: therapeutic opportunities. J Clin Invest 2013;123:966-72. https:// doi.org/10.1172/JCl64098 